\title{
Manejo de un central deciduo retenido por trauma dental: a propósito de un caso
}

\author{
Management of a central deciduous tooth retained by dental trauma: A case study \\ Orlando Martínez-Bermúdez ${ }^{*}$ orcid.org/0000-0002-5347-1039 \\ Jennifer Orozco-Páez1 orcid.org/0000-0003-4834-071X \\ Vivi Hoyos-Hoyos ${ }^{2}$ orcid.org/0000-0002-3085-7772 \\ Johan García-Estaño3 orcid.org/0000-0002-6222-7492 \\ Nohora Salas-Molina ${ }^{3}$ orcid.org/0000-0002-1523-303X \\ Karina Cabrera-Jiménez ${ }^{3}$ orcid.org/0000-0002-8602-5297 \\ Jaime Plazas-Román' orcid.org/0000-0002-5040-6899
}

1. Docente del programa de Odontología, Corporación Universitaria Rafael Núñez. Cartagena de Indias, Colombia

2. Odontóloga, Corporación Universitaria Rafael Núñez. Cartagena de Indias, Colombia

3. Estudiante del programa de Odontología, Corporación Universitaria Rafael Núñez. Cartagena de Indias, Colombia central deciduo retenido por trauma dental: a propósito de un caso. Univ. Salud. 2021;23(1):71-75. DOI: https://doi.org/10.22267/rus.212301.216

\section{Resumen}

Introducción: El trauma dentoalveolar es un tipo de afección muy común en niños, que en la mayoría de los casos involucra dientes, la porción alveolar del maxilar, la mandíbula y los tejidos blandos adyacentes. Pueden variar en cuanto a la extensión y gravedad llegando a afectar la funcionalidad e integridad de las estructuras orales, por lo que un diagnóstico correcto y tratamiento oportuno es necesario para evitar la generación de posibles secuelas en la dentición permanente. Objetivo: Reportar un caso de trauma dentoalveolar en un niño y su abordaje odontológico interdisciplinar. Presentación del caso: Paciente masculino de 7 años de edad, sin antecedentes relevantes, asiste a consulta con su madre por presentar persistencia de un diente temporal en sector anterosuperior, asociado a trauma dentoalveolar ocurrido a los 18 meses de vida aproximadamente. Se realiza exodoncia del diente temporal y tratamiento ortopédico para orientar la erupción del diente permanente, manifestando evolución favorable. Conclusiones: El abordaje interdisciplinario para los casos de trauma dento-alveolar constituye una buena alternativa para una adecuada rehabilitación y disminuye posibles secuelas.

Palabras clave: Traumatismos de los dientes; diente deciduo; atención odontológica; odontología pediátrica; niños. (Fuente: DeCS, Bireme).

\begin{abstract}
Introduction: Dentoalveolar trauma is a common type of condition in children, which mostly affects the teeth, alveolar segment of the maxilla, mandibula and adjacent soft tissues. It can vary in terms of extension and severity, affecting the functionality and integrity of the oral structures. Therefore, a correct diagnosis and a timely treatment are necessary to avoid future sequelae in the permanent dentition. Objective: To report a case of dentoalveolar trauma in a child and its interdisciplinary dental approach. Case presentation: A 7-year-old male child with no relevant history attends a consultation with his mother due to the persistence of a temporary tooth in the anterosuperior sector. It is associated with dentoalveolar trauma that occurred when he was 18 months old. Exodontia and orthopedic treatment were performed on the temporary tooth to guide the eruption of the permanent tooth, showing a favorable evolution afterwards. Conclusions: An interdisciplinary approach for cases of dentoalveolar trauma constitutes an appropriate alternative for successful rehabilitation and reduces possible sequelae.
\end{abstract}

Keywords: Tooth injuries; tooth deciduous; dental care; pediatric dentistry; children. (Source: DeCS, Bireme).

\footnotetext{
*Autor de correspondencia

Orlando Martínez-Bermúdez

e-mail: orlandoemartinez@gmail.com
} 


\section{Introducción}

Los traumas dentoalveolares son lesiones que afectan dientes, hueso y tejidos de soporte, derivados de eventos secundarios como accidentes o de forma intencional que puede provocar daño a nivel funcional, psicológico y social ${ }^{1}$. Se estima que el $80 \%$ de las lesiones ocurren en personas menores de 20 años, con predominio en el género masculino. La presencia de maloclusiones y la condición socioeconómica del paciente son factores que se han considerado predisponentes para su desarrollo ${ }^{2}$.

El trauma dentoalveolar se ha convertido en un problema de salud pública debido a su alta incidencia, especialmente en la población infantil. De acuerdo a los estudios epidemiológicos reportados, los dientes afectados con mayor frecuencia son los anterosuperiores, siendo los incisivos centrales los que presentan mayor compromiso ${ }^{3}$. La identificación y el tratamiento de los traumas en dentición primaria es muy relevante para evitar la generación de posibles secuelas o alteraciones en la dentición permanente, considerando factores como: la edad del paciente, la gravedad de la lesión, la cercanía del ápice del diente afectado con el germen del diente sucedáneo, la conservación de la tabla alveolar, la presencia de abscesos, entre otros, que son determinantes importantes para su evolución y posterior tratamiento, lo cual puede contribuir a mejorar las tasas de éxito a nivel terapéutico y/o restaurativo ${ }^{4,5}$.

Dependiendo de la magnitud del trauma, la lesión puede influir no sólo en la funcionalidad del sistema estomatognático, sino también, en el estado psicosocial del niño, ya que, los cambios físicos en algún momento terminan afectando el estado emocional del paciente y por ende su calidad de vida $^{6}$. De allí que el abordaje odontológico integral y oportuno de este tipo de lesiones cobra especial importancia, con el fin de garantizar la rehabilitación total del niño evitando al máximo secuelas. En este sentido, el objetivo de este artículo es reportar un caso de trauma dentoalveolar en un niño y su abordaje odontológico.

\section{Descripción del caso}

Paciente masculino de 7 años de edad, asiste a consulta odontológica con su madre para revisión de rutina en la Clínica Odontológica de la Corporación Universitaria Rafael Núñez, sin antecedentes médico personales relevantes al caso. Al realizar un diagnóstico inicial por parte de semiología, la madre del menor refiere la persistencia de un órgano dental deciduo en el sector anterosuperior, así mismo, comenta que el menor sufrió un trauma en esta zona a la edad de 1 año y seis meses aproximadamente, que le generó sensibilidad al tacto en la zona afectada y movilidad dental (Grado 1 de acuerdo a lo descrito por la madre). Posterior a la anamnesis, se decide remitir al niño al área de odontopediatría.

Al realizar el examen clínico, se observó ausencia del Organo Dental 210D 21, en su lugar persistencia del OD 61 con destrucción coronaria y pólipo pulpar. Además, se identificó fístula en encía adherida vestibular a nivel de este mismo órgano dentario. $\mathrm{Su}$ contralateral OD 11 se observó totalmente erupcionado sin alteraciones (Figura 1). En el examen radiográfico se evidenció la presencia del OD 21 incluído, en un estadio de desarrollo de Nolla 8, ubicado en posición vertical y presencia del 61, el cual persiste en su posición conservando al menos un tercio de su raíz. No se observó fractura u otro tipo de afección al diente permanente (Figura 2).
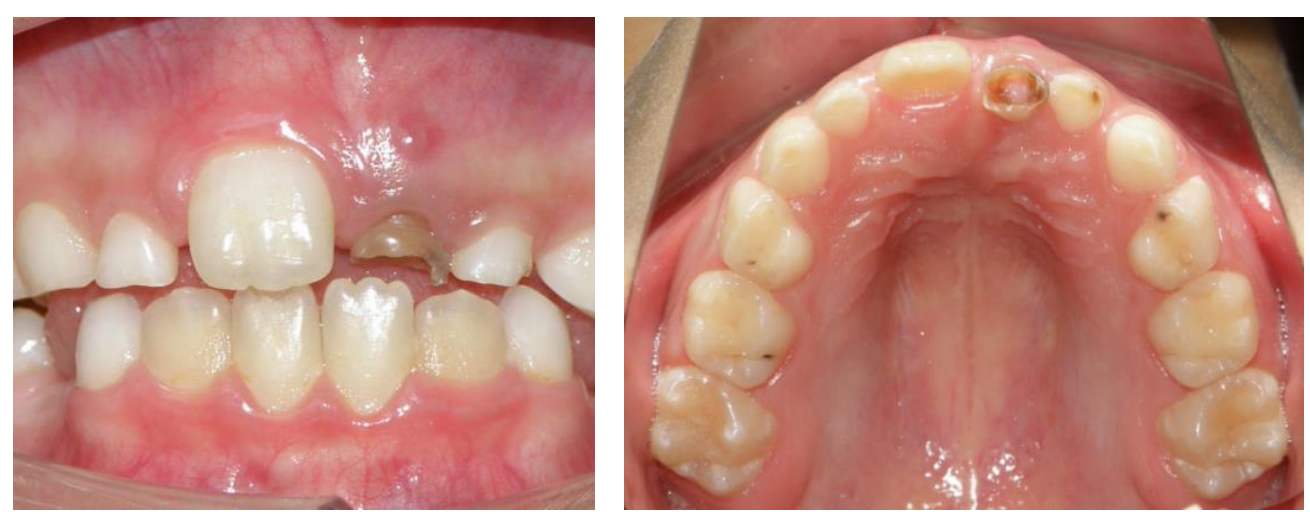

Figura 1. Fotografías intraorales iniciales. Se observa OD 61 persistente con fractura coronal y fistula a la altura del ápice. 


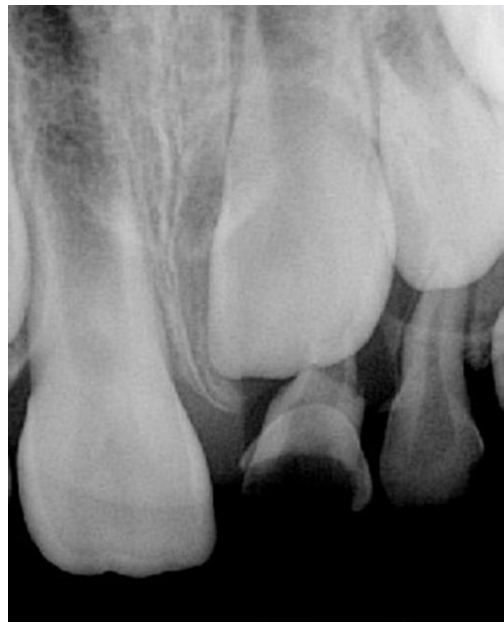

Figura 2. Radiografía periapical inicial.

Se realizaron interconsultas con especialistas de odontopediatría, ortodoncia y ortopedia maxilar. Teniendo en cuenta los hallazgos clínicos, el diagnóstico bucal establecido por el odontopediatra para el caso fue:

- Fractura corono - radicular con participación pulpar en el OD 61,

- Órgano dentario 21 incluido,

- Caries dental en OD 55 y 64,

- Gingivitis generalizada asociada a biopelícula.

Los diagnósticos del ortodoncista y ortopedia maxilar fueron:

- Perfil recto con competencia labial,

- Clase I de Angle y esquelética.

Las fases del tratamiento realizado incluyeron:

1. Fase Higiénica y preventiva: motivación, enseñanza de cepillado, profilaxis y aplicación de flúor en barniz.

2. Fase operatoria: operatoria dental en OD 55 y 64 .
3. Fase quirúrgica: se realizó exodoncia del órgano dentario 61, sin complicaciones.

Fase ortopédica: posterior al proceso de cicatrización fueron colocadas dos placas de Hawley con tornillo $(8 \mathrm{~mm})$ de expansión transversal para realizar una expansión dentoalveolar lenta de $1 \mathrm{~mm}$ por semana ( 2 giros cada tres días) y usadas como mantenedores de espacio para OD 74 y 84, se realizó control clínico y radiográfico mensualmente. Aproximadamente a los 4 meses de evolución se observó la erupción espontánea del órgano dental 21 (Figura 3).

Después del control realizado a los 4 meses, el paciente abandonó la consulta debido a que era de origen extranjero. En la última cita se le dieron indicaciones al paciente y a la madre para realizar la activación de la aparatología ortopédica dando 2 giros cada 15 días hasta alcanzar el límite de elongación del tornillo transversal de la placa, esto con el fin de que el OD 21 siguiera con el proceso de erupción que había iniciado. Asimismo, se le hizo refuerzo en promoción de la salud y prevención de la enfermedad oral. Por lo cual, tanto el paciente como la madre refirieron sentirse satisfechos con los resultados obtenidos.

\section{Consideraciones éticas}

Este caso clínico contó con el aval del comité de ética por parte de la Corporación Universitaria Rafael Núñez. Se tuvo en cuenta las normas vigentes sobre investigación en seres humanos contempladas en la Resolución 8430 de 1993 del Ministerio de Salud de Colombia. Se explicó a los padres cada etapa del procedimiento y realizaron firma de consentimiento informado autorizando la revisión de su historia clínica y publicación de sus imágenes diagnósticas.
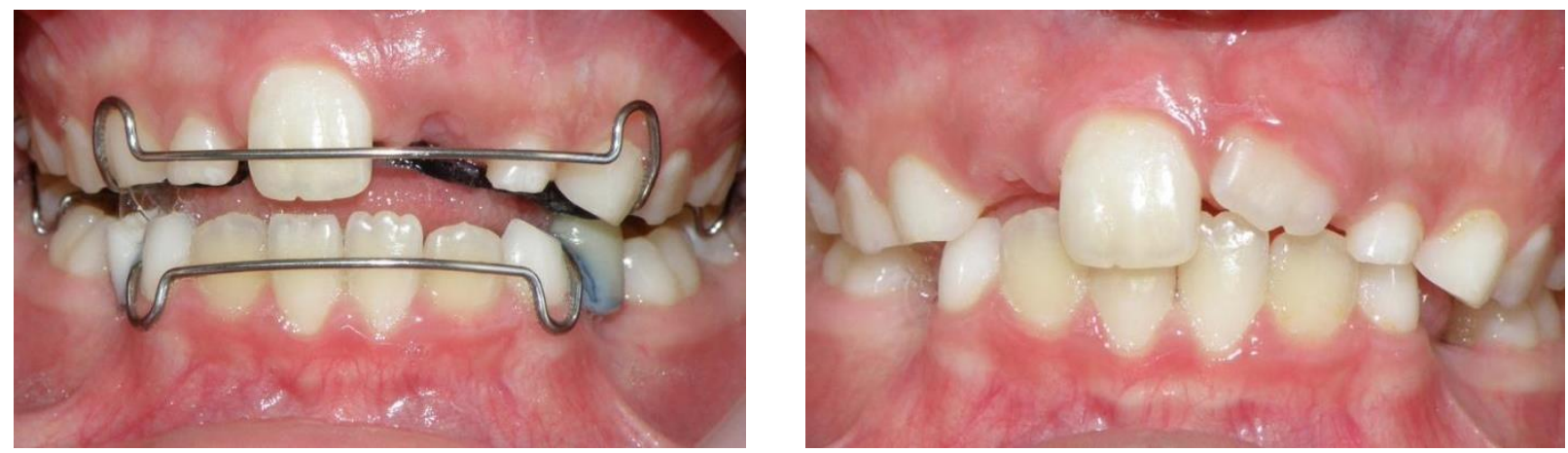

Figura 3. A. Colocación de placas de Hawley en maxilar superior e inferior, B. Evolución a los 4 meses, se observa erupción espontánea del OD 21. 


\section{Discusión}

El trauma dentoalveolar durante la infancia constituye una de las principales causas de consulta odontológica. Se estima que afecta aproximadamente al $30 \%$ de la población con dentición primaria. Gutiérrez et al. 7, indican que la principal causa son los accidentes, como colisiones contra objetos y/o personas, accidentes, caídas y actividades relacionadas con el deporte. Por su parte, Ritwik et al. 8, afirman que los dientes incisivos centrales superiores, seguidos por los incisivos laterales superiores son los órganos dentales afectados con mayor frecuencia. Batista et al. ${ }^{9}$, exponen que el trauma en estos dientes tiene una alta repercusión en las funciones de masticación y fonación, así como problemas estéticos.

El tratamiento de cada caso debe ser precedido por un minucioso examen clínico estomatológico y radiográfico ${ }^{3}$. El presente caso refleja un patrón de trauma reportado en la literatura como "sin complejidad"5; sin embargo, la adecuada evaluación de las características del paciente permitió que se realizara un abordaje conservador en lugar de un tratamiento invasivo como es el caso de la tracción ortodóntica, en la que se requiere intervención quirúrgica ${ }^{3}$.

Fueron varios los factores que se tuvieron en cuenta para la toma de decisiones, entre ellos: la edad del paciente, importante para establecer el tiempo de erupción en el que se encuentra el niño y el grado de desarrollo maxilar, en este caso el niño tenía 7 años y el OD 11 (contralateral del diente afectado) se encontraba erupcionado próximo a entrar en la línea de oclusión, lo que indicó que el periodo de erupción del OD 21 estaba próximo a terminar y el hecho de estar impactado podría alterar la cronología de la erupción de sus dientes vecinos, en este caso el OD 12. Otro factor evaluado es el estadio de Nolla del diente permanente, el cual fue favorable en este caso para la erupción espontanea del diente y que aún se encontraba en proceso de formación radicular; además se tuvo en cuenta el diagnóstico de la estructura dental a nivel y la distancia del borde incisal del OD 21 a la línea de oclusión de su antagonista, el cual se encontraba cerca, lo que fue favorable; todas estas variables fueron decisivas para establecer el plan de tratamiento interceptivo, en el cual se decidió realizar la extracción del diente temporal, para prevenir la impactación del permanente debido a la permanencia del órgano dental primario.

La adecuada evaluación del trauma, es determinante para el pronóstico del tratamiento que se ejecuta en los pacientes y una de las principales dificultades que presentan los estudios que evalúan dientes incluidos, retenidos e impactados, es la ausencia de consenso en la definición de estos términos, siendo común que en el concepto de impactación dental se mezclen estas entidades 10,11 . Sumado a lo anterior, en la mayoría de estos estudios no se toma en cuenta el estado de formación dental de los dientes evaluados, muy posiblemente debido a las dificultades que presenta la determinación del estadio de Nolla en radiografías panorámicas análogas 12,13 , con lo que se puede estar tomando un porcentaje de gérmenes dentales con posibilidades de emerger a la cavidad bucal, y los cuales no representan una anomalía dental ${ }^{14}$.

Estrada y Katagiri15, destacan la importancia de realizar un diagnóstico, un examen clínico y radiográfico intraoral preciso y un tratamiento adecuado de los dientes retenidos evitando así, futuras complicaciones funcionales $\mathrm{y}$ estéticas mediante un trabajo multidisciplinario evaluando el cuadro clínico y radiográfico, con un seguimiento que permita determinar los cambios producidos en el proceso.

El caso aquí descrito provee información en torno a la naturaleza y manejo de una lesión generada por trauma dentoalveolar en la dentición primaria, el cual es pertinente para el conocimiento del profesional de la odontología, se resalta el tratamiento con enfoque integral que conllevó al abordaje correcto evitando afectaciones en el diente sucedáneo.

\section{Conclusiones}

En casos de dientes impactados asociados a trauma dentoalveolar, es necesario estudiar las características clínicas y radiológicas del caso en aras de conocer el grado de compromiso del diente permanente y si el trayecto de erupción es favorable. Se deben tener en cuenta aspectos como: el tipo y tiempo de evolución del trauma dentoalveolar, la edad del paciente, el proceso de rizoclasia del diente deciduo y el nivel de formación radicular del diente permanente. El estudio de estos factores determinará la posibilidad de orientar la erupción 
espontánea del diente comprometido mediante el empleo de aparatología ortopédica maxilar o la necesidad de intervención quirúrgica y tracción ortodóntica obteniendo mejores resultados de rehabilitación en el paciente.

\section{Conflicto de intereses}

Los autores no declaran conflictos de intereses.

\section{Referencias}

1. Fernández Collazo ME, Rodríguez Soto A, Vila Morales D, Pérez Fuentes M, Bravo Seijas B. Características asociadas al trauma dentoalveolar en incisivos superiores. Rev Cubana Estomatol [Internet]. 2013 [fecha de acceso: 29 de enero de 2020];49(2):153-161. Disponible en: http://scielo.sld.cu/scielo.php?script=sci_arttext\&pid=S003 4-75072013000200003\&lng=es.

2. Goettems ML, Brancher LC, da Costa CT, Bonow MN, Romano AR. Does dental trauma in the primary dentition increases the likelihood of trauma in the permanent dentition? A longitudinal study. Clin Oral Investig. 2017;21(8):2415-2420. doi: 10.1007/s00784-016-2037-3.

3. Turkistani J, Hanno A. Recent trends in the management of dentoalveolar traumatic injuries to primary and young permanent teeth. Dent Traumatol. 2011;27(1):46-54. doi: 10.1111/j.1600-9657.2010.00950.x.

4. Hernández Hernández E, Varga Servin L, Medina Solís C, Varela Ibañez C, Anton Baños M. Manejo inmediato de intrusión dental y lesión en tejidos blandos: Reporte de caso. Rev Estomatol Hered [Internet]. 2015 [fecha de acceso: 29 de enero de 2020];25(3):218-223. Disponible en: http://www.scielo.org.pe/scielo.php?script=sci_arttext\&pid $=$ S1019-43552015000300007

5. Andreasen JO, Lauridsen E, Gerds TA, Ahrensburg SS. Dental Trauma Guide: a source of evidence-based treatment guidelines for dental trauma. Dent Traumatol. 2012;28(5):345-50. doi: 10.1111/j.16009657.2011.01059_1.x.

6. Soto Carbelo LA, Curbelo Mesa R, Torres Sarma L. Frecuencia de traumatismos dentales en los incisivos en niños de 6 a 12 años. Rev haban cienc méd [Internet]. 2016 [fecha de acceso: 29 de enero de 2020];15(1):101-112. Disponible en: http://scielo.sld.cu/scielo.php?script=sci_arttext\&pid=S172 9519X2016000100012\&lng=es

7. Marriaga Gutiérrez A, Navarro Jiménez E, Barrios Angulo Á, De León Mercado P, Hernández Rojas G, Lasprilla Fawcett S. Evaluación del conocimiento de tratamiento de trauma dentoalveolar entre odontólogos de hospitales públicos en Barranquilla, Colombia. Salud Uninorte. 2018;34(1):58-67. doi: 10.14482/sun.34.1.9586

8. Ritwik P, Massey C, Hagan J. Epidemiology and outcomes of dental trauma cases from an urban pediatric emergency department. Dent Traumatol. 2015;31(2):97-102. doi: 10.1111/edt.12148.

9. Batista Sánchez T, Tamayo Ávila JO, Soto Segueo M, Paz Gil L. Traumatismos dentarios en niños y adolescentes. CCM [Internet] 2016 [fecha de acceso: 29 de enero de 2020];20(4):741-756. Disponible en: http://scielo.sld.cu/scielo.php?script=sci_arttext\&pid=S156 043812016000400012\&lng=es

10. Patil S, Maheshwari S. Prevalence of impacted and supernumerary teeth in the North Indian population. J Clin Exp Dent. 2014;6(2):e116-20. doi: 10.4317/jced.51284.

11. Topkara A, Sari Z. Investigation of third molar impaction in Turkish orthodontic patients: Prevalence, depth and angular positions. Eur J Dent. 2013;7(1):S94-S98. doi: 10.4103/1305-7456.119084.

12. Costa VPP, Goettems ML, Baldissera EZ, Bertoldi AD, Torriani DD. Clinical and radiographic sequelae to primary teeth affected by dental trauma: a 9-year retrospective study. Braz Oral Res. 2016;30(1):e89. doi: 10.1590/18073107BOR-2016.vol30.0089.

13. Mendoza Mendoza A, González Mallea E, Iglesias Linares A. Intrusive luxation in primary teeth: A Case Report. J Clin Pediatr Dent. 2015;39(3):215-8. doi: 10.17796/1053-462839.3.215.

14. Cagetti MG, Marcoli PA, Berengo M, Cascone P, Cordone L, Defabianis $\mathrm{P}$, et al. Italian guidelines for the prevention and management of dental trauma in children. Ital J Pediatr. 2019;45(1):157. doi: 10.1186/s13052-019-0734-7.

15. Estrada Manilla A, Katagiri Katagiri M. Tratamiento ortodóntico-quirúrgico de incisivo central impactado. Rev mex ortod. 2017;5(3):190-195. doi: 10.1016/j.rmo.2017.12.0 